\title{
Abstracts presented at the South West ENT Academic Meeting 2015, Bath, UK
}

\author{
A spontaneous pneumocephalus and \\ hyperpneumatisation of occipital bone and atlas \\ vertebra: a case report
}

R Sawant, A Trinidade, T Das
From the Addenbrooke's Hospital, Cambridge

\section{Introduction}

Pneumocephalus is the presence of air or gas within cranial cavity. It is caused by craniofacial trauma, tumours of skull base, after neurology or otolaryngology surgeries, occasionally noted in scuba diving and rarely it is spontaneous. Rarely, there can be hyperpneumatisation of skull as well as vertebra, a rare benign condition which can be related to elevated middle-ear pressure.

\section{Case report}

A 62-year-old fit and healthy gentleman presented with sudden, severe headache without any history of head injury or surgery. He did not have any focal neurological signs. On computed tomography (CT) scan of the head, he was found to have pneumocephalus and hyperpneumatisation of occipital bone and atlas vertebra. There was no obvious tegmen defect on CT scan. Therefore, myringotomy and grommet insertion was done to help decompression of pneumocephalus. He symptomatically improved after surgery and analgesia.

\section{Discussion}

Spontaneous pneumocephalus is a rare condition. Only 24 cases are reported with spontaneous onset of pneumocephalus. It should be considered even if there is no focal neural deficit. Hyperpneumatisation of skull and vertebra is a rare condition as well. It is embryological developmental anomaly. There are 125 such cases reported, but only 5 of them are related with spontaneous pneumocephalus. One condition could lead to other, which is discussed only in two articles. Most of the pneumocephalus cases were treated with surgery to repair the dural defect; only two cases were managed conservatively. We have treated our case with minimal surgical intervention to decompress the pneumocephalus.

Paediatric inflammatory neck abscess: who is likely to undergo surgical drainage?

Y Kamhieh, N Doddi

From the Royal Gwent Hospital, Newport, Wales

\section{Introduction}

Inflammatory cervical lymphadenitis is common in the paediatric population. Some cases progress to abscess formation. In this antibiotic era, small abscesses are being treated conservatively. However the criteria to determine who will need surgical drainage have not been well established. Our study aimed to determine the factors that would necessitate surgical drainage.

\section{Methods}

This is a retrospective study of all children aged up to 16 years with ultrasound diagnosis of cervical lymph node abscess, from 2004 to 2014. Suspected cold abscesses and non-lymph node abscesses were excluded. In total, 34 patients had a confirmed 'hot abscess'; 24 patients (70 per cent) required surgical excision.

Results

Comparing patients treated conservatively or surgically yielded no significant difference in gender distribution $(p=0.7)$, mean age $(p=0.5)$, inflammatory markers or length of admission. However, abscess size approached a significant difference; $21 \mathrm{~mm}$ in patients managed conservatively versus $27 \mathrm{~mm}$ in patients requiring surgery ( $p=$ 0.095). Abscesses bigger than $10 \mathrm{~mm}$ were nine times likelier to necessitate surgery (odds ratio $=9, p=0.075$ ).

\section{Conclusion}

We propose that there is a quantifiable abscess size, below which non-surgical therapy is preferable. Our preliminary results reflect our experience with lymph node abscesses over 10 years, and suggest that the likelihood of successful medical management is lower if the abscess size exceeds $10 \mathrm{~mm}$.

Assessment of laryngectomy practice in the South West Region and a proposal for standard setting for laryngectomy practice

S Hollis $^{1}$, S A Burrows ${ }^{1}$, D L Baldwin ${ }^{2}$, W O Bennett ${ }^{3}$, D Black $^{4}$, G Barrett $^{5}$, S Al-Zahid ${ }^{6}$, E Toll ${ }^{7}$, V Reddy ${ }^{8}$

From the ${ }^{1}$ Gloucester Royal Hospital, ${ }^{2}$ North Bristol NHS Trust, ${ }^{3}$ Musgrove Park Hospital, Taunton, ${ }^{4}$ Royal United Hospital Bath, ${ }^{5}$ Royal Devon and Exeter Hospital, Exeter, ${ }^{6}$ Torbay Hospital, Torquay, ${ }^{7}$ Derriford Hospital, Plymouth, and ${ }^{8}$ Royal Cornwall Hospital, Truro

\section{Introduction}

With the introduction and advancement of radio-oncological techniques the number of laryngectomy procedures has decreased. The reduction of numbers has raised questions regarding the maintenance of standards and training. The government has proposed that there should be centralisation of major head and neck services. The South West is a very spread-out region with a comparatively sparse population in comparison to other areas of England and Wales. We undertook an appraisal of laryngectomy practice and outcomes around the region to look at possible variations in outcomes and to propose standards for practice. 


\section{Methods}

A retrospective analysis of the 10 last laryngectomy procedures undertaken by each unit in the South West region. We assessed the spread of time that 10 were undertaken, their indications, the patient's pre-operative morbidity, surgical techniques, 30-day morbidity and mortality and oncological outcomes.

Results

It is apparent that there is wide variation in multidisciplinary team preference for surgery both as primary treatments and in recurrent disease. There was a range of $80 / 20$ per cent primary surgery to salvage to $80 / 20$ per cent the other way around. This significantly affected the patient's outcome. A multivariate analysis was performed that showed the need for transfusion and positive margins significantly affected outcome. All departments within the region had fistula rates comparable to those reported in the literature.

\section{Discussion}

Although the departments had outcomes that were comparable or better than those published, it was felt that we could propose standards to enable ongoing audit. We are in the early stages of setting up an ongoing regional audit to enable suitable comparisons and feedback to be given to the departments.

\section{Conclusion}

Despite the current drive for centralisation, in rural communities travel distances are an important consideration. Our region's departments all performed within expected standards drawn from the literature suggesting that departmental throughput may not have a significant bearing on outcome. More work is required.

Chronic rhinosinusitis and the sunshine vitamin: a systematic review

G Chawdhary ${ }^{1}$, R Schlosser ${ }^{2}$

From the ${ }^{1}$ Great Western Hospital, Swindon, UK and ${ }^{2}$ Medical University of South Carolina, Charleston, USA

\section{Introduction}

Vitamin D3 is classically known for its actions in bone and calcium homeostasis. Recently, it has also been shown to be a potent immunomodulatory steroid hormone, prompting studies on its potential role in chronic rhinosinusitis, especially chronic rhinosinusitis with nasal polyps, the eosinophilic, Th2-skewed form of the disease.

\section{Objective}

To review the literature on the role of vitamin D3 in chronic rhinosinusitis.

\section{Methods}

Systematic search of the following databases, limited to English language papers: Ovid Medline (1947 to July 2014), Embase (1974 to July 2014) and Cochrane Review databases. Reference lists of all identified studies were examined for further relevant papers.

\section{Results}

The literature on the role of vitamin D3 in chronic rhinosinusitis is limited to case series, case control and in vitro studies. A series of case control studies have found no significant difference in circulating vitamin D3 level between chronic rhinosinusitis without nasal polyposis and controls. In contrast, case control studies have found significantly lower vitamin D3 level in patients with chronic rhinosinusitis with nasal polyps than in controls. Additionally, in vitro studies have shown that chronic rhinosinusitis with nasal polyps epithelial cells have impaired ability to convert vitamin D3 into its active form, and the addition of vitamin D3 acts to suppress pro-inflammatory cytokines.

\section{Conclusion}

The current evidence suggests that low vitamin D3 level may play a role in the pathology of chronic rhinosinusitis with nasal polyps. Further work would seek higher-level evidence in this area and is important because, if proven, may open up a new therapeutic modality for this as yet incurable condition that of vitamin D3 supplementation.

\section{Clinical outcomes following surgical treatment of chronic parotid sialadenitis}

A Goomany, D Waterhouse, S Sood

From the Bradford Royal Infirmary

\section{Introduction}

Chronic parotid sialadenitis is an uncommon inflammatory disorder which can cause intractable symptoms and significant morbidity. Patients who fail conservative treatment may require surgical intervention. Controversy exists regarding the most appropriate pre-operative investigations and surgical management.

\section{Objective}

To analyse the clinical outcome of patients treated with parotidectomy for chronic sialadenitis.

\section{Methods}

A retrospective review of patients treated surgically for chronic parotid sialadenitis between 2007 and 2014. Seventeen patients (11 female, 6 male) were identified, of which 16 had a superficial parotidectomy and 1 total parotidectomy. Clinicopathological data, treatment morbidity and outcome were analysed.

\section{Results}

Average age at presentation was 52 years (range 19 to 72 years). Peri-prandial pain or swelling and parotid mass were the presenting features in 14 ( 82.4 per cent) and 3 (17.6 per cent) patients respectively. Pre-operative investigations included 10 ultrasound scans, 6 magnetic resonance imaging scans, 4 sialograms and 3 fine needle aspirations. Three patients had multiple imaging investigations. Average symptom duration was 7.28 months (range, 0.17 to 24), with no cases of pre-operative facial nerve palsy. Bilateral gland involvement occurred in three patients (two post-radioiodine therapy and one non-specific chronic inflammation). Nine patients ( 52 per cent) had complications (three with multiple complications), including Frey's syndrome (four patients), neuropathic pain (two), keloid (one), infection (one), seroma/haematoma (two), and collapse of the external auditory canal (one; total parotidectomy). Two (11.8 per cent) patients had House-Brackmann grade II facial nerve palsy at six months post-operatively. Complete symptom resolution was achieved in 16 (94.1 per cent) patients.

\section{Conclusion}

Superficial parotidectomy is an effective treatment for chronic parotid sialadenitis but has a high complication rate. 
How reliable is pre-operative computed tomography scanning in patients with chronic otitis media? A prospective, comparative review of otologists' and radiologists' predictive accuracy

A Mowat, R Kayarkar

From the Mid Yorkshire NHS Trust

\section{Introduction}

The development of high-resolution computed tomography (CT) represented a significant advance in temporal bone imaging. Its value lies in the detailed assessment of the erosion and obliteration of middle-ear structures.

\section{Objective}

This study assessed the accuracy of prospective predictions made by consultant radiologists and otologists, when compared with intra-operative findings.

\section{Methods}

Sixteen high-resolution CT scans were included. These were taken after 01 June 2013, by a single surgeon, before mastoidectomies, for chronic otitis media. Consultant radiologists, aware of the ongoing audit, routinely reported on these scans. Absence of comment was considered indicative of normal findings. The operating surgeon completed a proforma making 13 predictions; 6 were related to the erosion of the middle-ear structures (e.g. stapes) and 7 were related to anatomical variants (e.g. mastoid pneumatisation).

\section{Results}

Intra-operatively, cholesteatoma was confirmed in 81 per cent of cases. The incus was the most eroded ossicle $(88$ per cent), followed by the malleus (56 per cent) and stapes (31 per cent). The scutum was the most common, nonossicular, bony erosion (69 per cent). Comparatively, both groups had near identical predictive accuracy (otologists 90.6 per cent, radiologists 90.1 per cent). There was strong agreement on the position of the jugular bulb, dura and mastoid pneumatisation. There was poor agreement on erosion of the scutum, malleus and facial nerve dehiscence in the transverse plane.

\section{Conclusion}

There was no statistically significant difference between the predictive accuracy of consultant radiologists and otologists. High-resolution CT generally underestimated the extent of ossicular erosion and overestimated facial nerve dehiscence. Otologists should be prepared to encounter conditions not predicted by the radiologists, or themselves, and modify their strategy accordingly.

\section{Accuracy of drain readings by surgical healthcare professionals}

\author{
S Shankla, T Biggs, H Cox, E King \\ From the Poole General Hospital and the Royal Bournemouth \\ Hospital
}

\section{Introduction}

Redivac drains are commonly used in a variety of ENT procedures. It is standard practice for these to be removed when drainage reaches $\leq 30 \mathrm{ml} /$ day; therefore, accurate reading is essential. Underestimation increases infection risk and pain, and impacts upon hospital admission duration, which is a significant factor with the National Health Service facing intense finical pressures.

\section{Objective}

Our aim was to assess the ability of surgical healthcare workers to read simulated drain volumes, utilising both the main $(>50 \mathrm{ml})$ and corner measurement $(\leq 50 \mathrm{ml})$ scales.

\section{Methods}

Drains were injected with $25 \mathrm{ml}$ and $60 \mathrm{ml}$ aliquots of red wine (simulating blood) and milk (simulating chyle). Users were asked to read drains and were observed for technique and accuracy.

Results

In total, 30 healthcare workers were assessed (doctors and nurses of varying grades). The median assessment for the $25 \mathrm{ml}$ simulated blood drain was $45 \mathrm{ml}$ (range of 10 to $60 \mathrm{ml}$ ). Only one person correctly identified the volume as $25 \mathrm{ml}$. Twenty-five of 30 healthcare workers assessed the volume of the $25 \mathrm{ml}$ simulated blood drain bottle to be $\geq 30 \mathrm{ml}$.

\section{Conclusion}

This study has revealed a large inaccuracy in the reading of these simple and commonly used drains. Indeed, the majority of healthcare workers (25 out of 30) overestimated the simulated $25 \mathrm{ml}$ blood volume, which would result in an increased hospital stay if occurring in a clinical setting. Furthermore, the majority of users were unaware of the smaller scale and its use. This study has highlighted a need for further education on the methods of accurate drain assessment.

Cervical fine needle aspiration cytology: how reliable is it at diagnosing lymphoma?

M Cherko, A Salem, M Millip, J Ahmed, K Ghufoor

From the Royal London Hospital

\section{Introduction}

Fine needle aspiration cytology forms an integral part of the diagnostic panel used in the head and neck clinic. However, like many tests it can yield false results, and in the case of cancer this can create difficult challenges in patient management.

\section{Objective}

To determine the positive predictive value of cervical fine needle aspiration cytology in diagnosing lymphoma, and detail cases that do not correlate with histology.

\section{Method}

A retrospective analysis was performed of 150 consecutive cases of cervical fine needle aspiration cytology reporting lymphoma in our head and neck unit. Cytology results were compared with eventual histology diagnosis.

\section{Results}

We found a 74 per cent positive predictive value of cytology for lymphoma. Of the remainder, 9.3 per cent were reported as reactive lymph nodes, 12 per cent were other nonmalignant pathologies and 4.7 per cent were other malignant pathologies.

\section{Conclusion}

We found that there were a significant number of nonlymphoma pathologies diagnosed from tissue biopsies in cases reported as lymphoma from cervical fine needle aspiration cytology. Notably, almost 1 in 20 cases in the group 
were eventually diagnosed with a non-lymphoma malignancy. This is crucial information needed to provide adequate patient care, especially when the optimal management differs significantly from that for a diagnosis of lymphoma. Therefore, head and neck clinicians must be vigilant to these discrepancies and act to swiftly confirm diagnosis with tissue biopsies.

Extra-capsular dissection for benign parotid tumours: our experience in 100 patients

M Daoud, H Merai, K Ramachandran, N Morgan

From the Glangwili General Hospital, Carmarthen, Wales

\section{Introduction}

For decades, superficial parotidectomy has been widely accepted as the 'gold standard' procedure for benign parotid tumours because of low recurrence rates (2 per cent). However, studies have reported a higher incidence of temporary (8.8-76 per cent) and permanent facial palsy (0-14 per cent), questioning the necessity to remove the entire superficial lobe. More recently, studies have shown significantly reduced rates of temporary and permanent facial palsy with extra-capsular dissection. Extra-capsular dissection involves careful dissection around the tumour capsule without prior identification of the facial nerve.

\section{Objective}

To report outcomes of extra-capsular dissection for benign parotid tumours in a single centre and compare results with published data.

\section{Method}

Our cohort consisted of the first 100 patients who underwent extra-capsular dissection parotid surgery at Glangwili General Hospital between April 2004 and August 2013, of whom 41 were female and 59 male, with a mean age of 54. All masses were clinically benign, and investigations revealed no features suggestive of malignancy. A modified Blair incision was used in all cases, and intra-operative facial nerve monitoring was performed.

\section{Results}

None of the patients developed a permanent facial palsy. One developed a temporary partial facial palsy (involving the marginal mandibular nerve), which recovered completely within 20 months. None of the 39 pleomorphic adenoma patients have developed recurrence so far. Six developed a haematoma but only one needed surgical evacuation. There was one case each of: Frey's syndrome, sialocele and periauricular numbness.

\section{Conclusion}

Extra-capsular dissection of parotid tumours should be advocated for discrete, mobile masses less than $4 \mathrm{~cm}$, as our study, in addition to others, demonstrates a decreased incidence of facial palsy.

\section{An algorithmic approach to branchial cysts in the over forties}

H Turner, K Shah, S Winter

From the Oxford University Hospitals NHS Trust

\section{Introduction}

Branchial cysts usually present as a cystic lateral neck mass. However, with increasing age, cystic metastasis to nodes, predominantly from primary head and neck cancers remains a differential. It is therefore imperative to exclude malignancy prior to surgical excision; however, no clear guidelines exist for this patient group.

\section{Objective}

To propose an algorithm for clinicians seeing patients with lateral cystic neck lumps.

\section{Method}

Retrospective case note analysis of all histologically confirmed cases of branchial cysts excised between 2007 and 2014 at Oxford University Hospitals. A literature review was undertaken alongside the results of this study.

\section{Results}

Twenty-one patients were included in the study, with an average age of 52 years. Ninety-five per cent of patients (20 out of 21) had fine needle aspiration cytology. All 21 patients underwent pre-operative imaging with at least 1 of the following modalities: ultrasound scan, computed tomography $(\mathrm{CT})$, magnetic resonance imaging and positron emission tomography (PET)-CT. The majority of patients under the care of an otolaryngologist underwent examination of the aero-digestive tract under general anaesthetic prior to excision of the branchial cyst. Frozen section analysis of the neck mass at the time of surgery was performed in 48 per cent of patients (10 out of 21)

\section{Conclusion}

We have identified many approaches to diagnosis and treatment of these patients, and we propose a unifying algorithm of fine needle aspiration cytology and PET-CT scan. Surgically, we advocate a pan-endoscopy followed by excision biopsy of the mass. If malignancy is determined at any point, the algorithm is aborted and patients are referred to the multidisciplinary team.

Quantifying recurrent acute otitis media in children using a contemporary UK electronic health records cohort

M Verkerk ${ }^{1}$, H Evans ${ }^{1}$, S Denaxas ${ }^{2,3}$, H Blackshaw ${ }^{1}$, A Schilder ${ }^{1}$

From the ${ }^{1}$ EvidENT, University College London, Ear Institute, ${ }^{2}$ Farr Institute of Health Informatics Research, University College London and ${ }^{3}$ Institute of Health Informatics, University College London

\section{Introduction}

The incidence of recurrent acute otitis media in UK children is as yet unquantified, despite a known effect on quality of life comparable to asthma.

\section{Objective}

Using National Health Service electronic health records, we aimed to estimate the proportion of UK children that develop recurrent acute otitis media to enable future studies into the most effective management strategies for these children.

\section{Method}

We performed a retrospective analysis of a primary care electronic health records database ('CALIBER', Clinical Practice Research Datalink) from January 1997 to March 2010. Incident acute otitis media episodes in children aged less than 16 years old were defined using Read codes. Recurrent acute otitis media was defined by international 
consensus as: 3 or more episodes of acute otitis media in 6 months, or 4 or more episodes in 12 months.

\section{Results}

A total of 95699 children were identified as having their first acute otitis media recorded in the study period. Of these, 10594 children (11.1 per cent) received a second coded acute otitis media diagnosis within 6 months. For 1689 of these children (1.8 per cent), this acute otitis media episode represented the first episode in a period of recurrent acute otitis media as defined by international guidelines.

\section{Conclusion}

More than 1 in 10 UK children receive a second acute otitis media diagnosis within 6 months of a first episode. Almost 1 in 50 develop 'recurrent acute otitis media' (according to international criteria) within the next year. Electronic health records analysis offers the unique opportunity to estimate the burden of ENT conditions, but is currently underutilised. Our next step will link primary and secondary care data to study how management strategies (antibiotics, grommets, adenoidectomy) affect acute otitis media recurrence rates.

\section{Management of oro-antral fistulae - a team effort}

S Healy, V Singh, K Smart, S Mustafa

From the Royal Glamorgan Hospital, Llantrisant, Wales

\section{Introduction}

An oro-antral fistula is an abnormal communication between the maxillary sinus and oral cavity. It typically occurs following dental extraction, and is managed by dentists or maxillo-facial surgeons. Oro-antral fistula also presents after infection and radiotherapy. Patients complain of fluid entering the nose when drinking and a sensation of air rushing into the tooth socket. Diagnosis is made on examination and imaging. Pain and discharge suggests secondary sinusitis.

One study of 86 patients with oro-antral fistula found 100 per cent had antral infection, and 49 per cent had polyps or mucosal thickening in the maxillary sinus. Defects greater than $5 \mathrm{~mm}$ diameter or present for more than three weeks are referred to our maxillo-facial colleagues for surgical closure. Techniques include buccal flaps, palatal flaps and a buccal fat pad. It is recognised that management of coexistent sinus disease is important. The use of antibiotics and nasal decongestants is well established, and some studies have used antral washouts in addition to flap closure.

\section{Case report}

A series of four cases of oro-antral fistula associated with chronic maxillary sinusitis will be presented with computed tomography imaging. Each patient underwent a single procedure with simultaneous oral closure of the oro-antral fistula performed by a maxillo-facial consultant and functional endoscopic sinus surgery (FESS) performed by an ENT consultant. At follow up, all patients achieved successful closure of their oro-antral fistula.

\section{Discussion}

Although not routinely seen in ENT clinics, maxillary sinusitis is a common finding in oro-antral fistula. Our experience suggests a combined procedure with FESS is an effective approach to managing persistent cases of oro-antral fistula.
Who is the ENT acute clinic run by best?

A Mowat, V Ward

From the Mid Yorkshire NHS Trust

\section{Introduction}

The ENT emergency service varies significantly between trusts. There is no 'gold standard'. Nationally, a majority of acute clinics are Senior House Officer led. Most Senior House Officers are in their first ENT job, and begin running clinics without training.

\section{Objective}

Mid Yorkshire Trust is relatively unique in conducting staff grade registrar led clinics. The objective was to establish whether this is justified.

\section{Methods}

A retrospective notes audit of 100 patients was conducted between 01 June 2014 and 31 August 2014. The electronically documented clinic letter was used to ascertain the relevant information. A subjective judgement was made in each case as to whether the consultation could have been conducted by a Senior House Officer with the knowledge and practical skills required to pass both components of the Diploma in Otolaryngology - Head and Neck Surgery examination.

\section{Results}

Sixty per cent of appointments were otological and 36 per cent were rhinological. Fifteen per cent required the interpretation of an audiogram, 1 per cent needed admission and in two cases magnetic resonance imaging scans were ordered. Thirty-seven procedures were performed, 36 of which could have been performed by a competent Senior House Officer. Despite registrar review, follow-up rates were high (48 per cent). Important diagnoses were made following investigation, including a rhinological malignancy and necrotising otitis externa.

\section{Conclusions}

The majority of the service ( 82 per cent) can be safely run by a Senior House Officer, with appropriate senior and nursing support. The acute clinic is also an excellent training facility. However, it is inappropriate for Senior House Officers, new to the specialty, to be expected to run the acute service without thorough induction including practical skills. A prolonged period of observation is suggested, before new Senior House Officers are left alone to lead the acute service.

A survey to assess the availability and use of YouTube as an educational resource

D Black, A Jardine

From the Royal United Hospital, Bath

\section{Introduction}

YouTube is generally regarded as not work-appropriate and is often blocked from hospital computers. We propose that YouTube contains a large amount of useful medical content, although the quality is variable and could be improved.

\section{Objective}

Firstly, to demonstrate that YouTube contains content of educational value and should be more available in hospital. 
Secondly, that there is a need to collate high-quality material into an accessible peer-reviewed format.

\section{Method}

A questionnaire was constructed to assess attitudes towards YouTube and its current use in a district general hospital. This was distributed to doctors and other healthcare professionals using pre-existing hospital email lists.

Results

Responses were collected from 107 doctors and 176 other healthcare professionals. A large majority $(75.5$ per cent doctors, 68.6 per cent other) already used YouTube for their own education. A significant minority referred patients to YouTube (24.5 per cent doctors, 19.3 per cent other). Most responders had encountered problems accessing YouTube in hospital (81.4 per cent doctors, 72.4 per cent other). An even greater majority thought a peer-reviewed YouTube channel would be beneficial (95.2 per cent doctors, 94.6 per cent other).

\section{Conclusion}

YouTube is used extensively as an educational resource. It is currently difficult to access in hospital. Previous research found variability in the quality of medical videos on YouTube. A peer-reviewed channel would address this and provide a single platform from which to access the best quality videos. The authors are in discussions with management to make access to YouTube more widely available in hospital as well as proposing to YouTube the equivalent of a Google Scholar service. 